\title{
LATTICE GAS ANALOGUE OF SK MODEL: \\ A paradigm for the glass transition
}

\author{
Francesco M. RUSSO \\ Dipartimento di Fisica,Università di Roma Tor Vergata, \\ Via della Ricerca Scientifica 1, 00133 Roma, Italy \\ and \\ INFN, Sezione di Roma
}

February 10, 1998

\begin{abstract}
We investigate the connection between the well known SherringtonKirkpatrick Ising Spin Glass and the corresponding Lattice Gas model by analyzing the relation between their thermodynamical functions. We present results of replica approach in the Replica Symmetric approximation and discuss its stability as a function of temperature and external source. Next we examine the effects of first order Replica Symmetry Breaking at zero temperature. We finally compare SK results with ours and suggest how the latter could be relevant to a description of the structural glass transition.
\end{abstract}

To appear in Journal of PhysicsA

ROM2F $/ 97 / 44$ 


\section{Introduction}

It is well known[1] that Ising Model is equivalent to Lattice Gas, a system defined in terms of occupation variables $\tau$ taking values 0 and 1 . The Lattice Gas effective Hamiltonian is formally identical to Ising one[1]. A simple change of variables $(\sigma=2 \tau-1)$ maps each of the two Hamiltonians into the other, provided that Ising external field is related to lattice gas chemical potential by $h-J=\frac{1}{2} \mu$, where $J$ is Ising spin-coupling related to lattice gas site-coupling $\Phi$ by $J=\frac{1}{4} \Phi$. This results in a simple relation between Ising free energy density and Lattice Gas pressure: $p=h-\frac{1}{2} J-f$. The two systems have therefore the same phase diagram and the same critical behaviour (real gases and Ising magnets are in the same universality class).

For random systems[2] this whole argument breaks down because the relation between chemical potential and magnetic field involves the quenched couplings. As we shall see in the next sections this results in new and unexpected features for the phase diagram of the system.

For Neural Networks this inequivalence between $\operatorname{spin}( \pm 1)$ and occupation $(0,1)$ variables had already been pointed out and analyzed, see for example [3] and references therein.

Recently much effort has been devoted to develop a description of the structural glass transition $[4,5,6]$ within the framework of disordered systems. Much of these models were however based on Ising Spin variables instead of Lattice Gas ones which should be more appropriate for a condensed matter system. For disordered systems the two kinds of variables are not equivalent. To have a comparison term it would be useful to analyze the properties of a mean field disordered lattice gas model. This could also show us how the structural glass transition may be described in the same way as the liquid-gas one.

In section 2 we present our model and in the following one we analyze its ground state. In section 4 we discuss its relation with the SK model. Section 5 is dedicated to writing down the saddle point equations of the replica approach. In section 6 we analyze the low temperature behaviour of the Replica Symmetric approximation and show its Phase Diagram. A condensed version of section 5 and 6 was originally presented in ref. [7]. In section 7 we discuss the stability of the Replica Symmetry and look for the AT line of the model. Section 8 contains some preliminary results with broken Replica Symmetry and in section 9 we present a comparison of our 
main results with the SK's ones and draw some tentative conclusions.

\section{The Model}

We consider a system of $N$ sites. An occupation variable $\tau_{k}$, defined in each site $k$, can take the values 0 or 1 . The Hamiltonian of the system is taken to be formally identical to the SK's one $[9,10]$. The interaction energy between two different ( $k$ and $l$ ) occupied sites is taken to be $\phi_{k l}$ and the system is coupled to some external source $g$. The total effective Hamiltonian is therefore:

$$
H_{\phi}[\tau]=-g \sum_{k=1}^{N} \tau_{k}-\frac{1}{2} \sum_{l \neq k} \phi_{k l} \tau_{k} \tau_{l} .
$$

In magnetic language $g$ would be the external field, while for a Lattice Gas $g$ is the sum of the chemical potential, the kinetic contribution, and eventually an external force term.

The infinite-ranged interaction energies $\left\{\phi_{k l}\right\}$ are taken to be quenched independent Gaussian variables with zero mean and variance $\Phi^{2} / N$. This means that the model should not have, on average, a preference between an ordering transition in two sublattices, as in binary alloys, and gas-liquid transition. For the low-temperature behaviour we could expect al least two regimes. For a strong enough negative $g$ occupied sites should be energetically penalized and we expect, for most of the realizations of the noise, the ground state energy to be zero and the low temperature behaviour to be similar to that of a pure system. On the other hand, for a strong enough positive $g$ occupied sites should be energetically favoured and we could expect the low temperature behaviour of the system to be much similar to that of SK's one. In the following we shall take $\Phi$ as the unit of energy and set $\Phi \equiv 1$, so we have:

$$
P\left(\phi_{l k}\right)=\left(\frac{N}{2 \pi}\right)^{\frac{1}{2}} \exp \left[-\frac{1}{2} N \phi_{l k}^{2}\right] .
$$

Each $\phi_{k l}$ is taken to be equally distributed and therefore each site interacts with each other. We also set the Boltzmann constant equal to 1 , as a consequence $T, H, g$ and $\phi$ are all dimensionless.

For a given realization of the $\phi$ 's the partition function is:

$$
Z_{\phi}(\beta ; g)=\sum_{\{\tau\}} e^{-\beta H \phi[\tau]} .
$$


For a Lattice Gas this would be the Grand Canonical partition function, because the total number of occupied sites $\sum_{k} \tau_{k}$ is not constrained to a specific value. Strictly speaking we should call "pressure" the thermodynamic potential $(\ln Z)$, while the grandcanonical independent variable should be the chemical potential. To have a more immediate comparison with SK model, and also with other models based on spin variables, we shall however abusively refer to the thermodynamical variables as if they were the canonical ones. In the following we shall therefore call "free energy density" the thermodynamic potential and refer to $g$ as the "external field".

We are interested, as usual when dealing with quenched disorder, to evaluate the averaged free energy density:

$$
f(\beta ; g)=-\frac{1}{\beta N} \int P[\phi] \ln Z_{\phi} d \phi
$$

this will be done in the following using the replica approach $[2,8,9]$.

\section{$3 \quad$ Ground State Properties}

We now discuss in more detail the ground state picture which was conjectured in the previous section. First of all we observe that the energy of the configuration without occupied sites is zero for all realizations of the quenched couplings. We can therefore conclude that the ground state energy is always non-negative. Secondly, we can easily see that the configuration with all occupied sites has an average energy equal to $-g N$, which is exactly the same value obtained for the all-spins-up configuration of SK model. Combining these two results we obtain, for the (quenched averaged) ground state energy density, the following upper bound:

$$
u \leq-g \theta(g)
$$

A lower bound can be obtained from the knowledge of the eigenvalues for a large Gaussian random matrix. Let us call $\omega$ the maximum eigenvalue of the matrix $\left[\phi_{l k}\right]$. For every configuration we can write

$$
\sum_{l k} \tau_{l} \phi_{l k} \tau_{k} \leq \omega \sum_{k}\left(\tau_{k}\right)^{2}
$$


In our case we have $\left(\tau_{k}\right)^{2}=\tau_{k}$, and $\omega=2$ with probability one in the thermodynamic limit. Putting all together we obtain, for the Hamiltonian (1), the following bound:

$$
-(1+g) \sum_{k} \tau_{k} \leq H_{\phi}[\tau] .
$$

Taking the minimum of both sides we finally get:

$$
-(1+g) \theta(g+1) \leq u .
$$

We thus see that for $g \leq-1$ the upper and lower bounds saturate and $u=0$ with probability one in the thermodynamic limit. The ground state configuration is that with no occupied sites. Each configuration with a finite number of occupied sites has the same average energy density in the thermodynamic limit. We therefore conclude that the low temperature behaviour of the system should be very similar to that of a pure one.

On the other hand, for positive $g$ we have $u \leq-g$, bound which is saturated in the limit $g \rightarrow \infty$. This implies that our system has a (first order?) zero-temperature transition at some $g_{0}$ between -1 and 0 . We also speculate that for a large positive $g$ the low temperature behaviour of the system should approximate that of SK model. We note that in SK case repeating the previous arguments we would obtain $-1-|h| \leq u \leq-|h|$.

The lower limit for $g_{0}$ can be improved using Derrida's argument[11]. Let us define $\Sigma(g, N)$ the average number of configurations with negative energy for a system with $N$ sites:

$$
\Sigma(g, N)=\int P[\phi] \sum_{\tau} \theta\left(-H_{\phi}[\tau]\right) d \phi .
$$

For a negative $g$ we obtain, in the thermodynamic limit,

$$
\sigma \equiv \lim _{N \rightarrow \infty} \frac{1}{N} \ln \Sigma(g, N)=\ln 2-g^{2} .
$$

If $g<-\sqrt{\ln 2}$ we see that $\sigma<0$ and therefore $\Sigma(g, N) \rightarrow 0$ in the thermodynamic limit. Following Derrida[11] we can conclude that for $g<-\sqrt{\ln 2}=$ -0.8326 there are almost surely no configurations of negative energy in the thermodynamic limit. Since there always is at least a configuration of zero energy, this must be ground state energy. We thus obtain the lower bound $g_{0} \geq-\sqrt{\ln 2}=-0.8326$ for the transition field at zero temperature. 


\section{Relation with SK model}

Before proceeding in our analysis we want to discuss the connections between the SK model[9] and the previously introduced one. We assume, as for the corresponding homogeneous models[1], that site variables are related by:

$$
\sigma=2 \tau-1 \quad \Longleftrightarrow \quad \tau=\frac{1}{2}(\sigma+1) .
$$

Substituting (5) into the Hamiltonian (1) we get:

$$
-H_{\phi}\left[\frac{\sigma+1}{2}\right]=\frac{1}{2} \sum_{k}\left(g+\frac{1}{4} \Phi_{k}\right)+\sum_{k}\left(\frac{1}{2} g+\frac{1}{4} \Phi_{k}\right) \sigma_{k}+\frac{1}{8} \sum_{k \neq l} \phi_{k l} \sigma_{l} \sigma_{k}
$$

where:

$$
\Phi_{k}=\sum_{l}^{l \neq k} \phi_{k l}
$$

We stress that (6) connects a homogeneous (site-independent) field system to a local-field one. In fact, defining the SK effective Hamiltonian for a site-dependent magnetic field:

$$
H_{J, h}^{(S K)}[\sigma]=-\sum_{k} h_{k} \sigma_{k}-\frac{1}{2} \sum_{k \neq l} J_{k l} \sigma_{l} \sigma_{k}
$$

we can write:

$$
H_{\phi}\left[\frac{\sigma+1}{2}\right]=-\frac{1}{2} \sum\left(g+\frac{1}{4} \Phi_{k}\right)+\frac{1}{4} H_{\phi, h[\phi]}^{(S K)}[\sigma],
$$

and the local magnetic field is correlated to the couplings by:

$$
h_{k}[\phi]=2 g+\Phi_{k} .
$$

The change of variables (5) maps our Hamiltonian (1) into an SK-like one, but correlation given by (9) is enough to destroy their equivalence. In fact, summing over configuration, we get for the partition functions:

$$
Z_{\phi}(\beta ; g)=\exp \left[\frac{1}{2} \beta \sum_{k}\left(g+\frac{1}{4} \Phi_{k}\right)\right] Z_{\phi}^{(S K)}\left(\frac{\beta}{4} ; h[\phi]\right]
$$


and thus the relation between the free energy densities of the two models leads to:

$$
f(\beta ; g)=-\frac{1}{2} g+\frac{1}{4} \int f_{\phi}^{(S K)}\left(\frac{\beta}{4} ; h[\phi]\right] P[\phi] d \phi .
$$

Our system is therefore equivalent to an SK-like one in which the magnetic field is a local random variable correlated with the couplings. SK's averaged free energy is not directly related to ours. Relation (5) is thus not useful in investigating thermodynamics of our model that is not reducible to something known, we have to face it by itself.

\section{The Replica Symmetric Solution}

Let us now proceed in applying replica formalism[2] to our system; we have to calculate the averaged $n$-th power of the partition function:

$$
Z_{n}=\overline{\left(Z_{\phi}\right)^{n}}=\int\left(Z_{\phi}\right)^{n} P[\phi] d \phi .
$$

For integer $n$ we get, after performing Gaussian integration:

$$
Z_{n}=\sum_{\{\tau\}} \exp \left[\sum_{k} \beta g \sum_{a} \tau_{k}^{a}+\frac{\beta^{2}}{2 N} \sum_{k<l}\left(\sum_{a} \tau_{l}^{a} \tau_{k}^{a}\right)^{2}\right]
$$

using the identity:

$$
2 \sum_{k<l}\left(\sum_{a} \tau_{l}^{a} \tau_{k}^{a}\right)^{2}=\sum_{a, b}\left(\sum_{k} \tau_{k}^{a} \tau_{k}^{b}\right)^{2}-\sum_{k} \sum_{a, b} \tau_{k}^{a} \tau_{k}^{b}
$$

which follows from the relation $\tau^{2}=\tau$, we can reorder the exponent and obtain:

$Z_{n}=\sum_{\{\tau\}} \exp \left[\beta \sum_{k}\left(g \sum_{a} \tau_{k}^{a}-\frac{\beta}{4 N} \sum_{a, b} \tau_{k}^{a} \tau_{k}^{b}\right)\right] \prod_{a, b} \exp \left[\frac{4}{N \beta^{2}}\left(\frac{\beta^{2}}{4} \sum_{k} \tau_{k}^{a} \tau_{k}^{b}\right)^{2}\right]$

Using Gaussian identities we rewrite (14) as:

$$
Z_{n}=\sum_{\{\tau\}} \exp \left[\beta \sum_{k}\left(g \sum_{a} \tau_{k}^{a}-\frac{\beta}{4 N} \sum_{a, b} \tau_{k}^{a} \tau_{k}^{b}\right)\right] \times
$$




$$
\times \prod_{a, b} \int\left(\frac{N \beta^{2}}{4 \pi}\right)^{\frac{1}{2}} \exp \left(-\frac{N}{4} \beta^{2} Q_{a b}^{2}+\frac{1}{2} \beta^{2} \sum_{k} Q_{a b} \tau_{k}^{a} \tau_{k}^{b}\right) d Q_{a b},
$$

reordering the exponentials and defining:

$$
\begin{gathered}
H_{Q}[\tau]=-\frac{1}{2} \beta^{2} \sum_{a, b}\left(Q_{a, b}-\frac{1}{2 N}\right) \tau^{a} \tau^{b}-\beta g \sum_{a} \tau^{a}, \\
A[Q]=\frac{\beta^{2}}{4} \sum_{a, b} Q_{a b}^{2}-\ln \left[\sum_{\{\tau\}} e^{-H_{Q}[\tau]}\right],
\end{gathered}
$$

we finally get:

$$
Z_{n}(\beta ; g)=\left(\frac{N \beta^{2}}{4 \pi}\right)^{\frac{n^{2}}{2}} \int e^{-N A[Q]} d^{n^{2}} Q
$$

The averaged free energy density is given by:

$$
f(\beta ; g)=\lim _{N \rightarrow \infty} \lim _{n \rightarrow 0}-\frac{1}{\beta n N} \ln Z_{n}(\beta ; g) \text {. }
$$

In the thermodynamic limit the integral can be estimated by maximizing the integrand, and this yields:

$$
f_{n}(\beta ; g)=\lim _{N \rightarrow \infty}-\frac{1}{\beta n N} \ln Z_{n}(\beta ; g)=\frac{1}{\beta n} \inf _{Q}\{A[Q]\}
$$

The extremum is determined from the saddle point equation:

$$
\frac{\partial A}{\partial Q_{a b}}=\frac{\beta^{2}}{2} Q_{a b}-\frac{\beta^{2}}{2} \frac{\sum_{\tau} \tau^{a} \tau^{b} e^{-H_{Q}[\tau]}}{\sum_{\tau} e^{-H_{Q}[\tau]}}=0,
$$

that may be rewritten as $Q_{a b}=\left\langle\tau^{a} \tau^{b}\right\rangle_{Q}$.

We first consider saddle points that are symmetric under the Replica Group[2]. Setting $Q_{a b}=q+b \delta_{a b}$ we can write $\sum_{a b} Q_{a b} \tau^{a} \tau^{b}=q\left(\sum_{a} \tau^{a}\right)^{2}+$ $b \sum_{a} \tau_{a}$ and $\sum_{a b} Q_{a b}^{2}=n(q+b)^{2}+n(n-1) q^{2}$, substitution in (17) and extraction of the $n \rightarrow 0$ limit then yields:

$$
f=\frac{1}{4} \beta b(2 q+b)-\left(2 \pi \beta^{2}\right)^{-\frac{1}{2}} \int_{-\infty}^{\infty} \ln \left[1+e^{\beta(\alpha+z \sqrt{q})}\right] e^{-\frac{1}{2} z^{2}} d z .
$$


In equation (22) we set $\alpha=g+\frac{1}{2} \beta b$, and the matrix elements satisfy the coupled equations:

$$
\begin{gathered}
\rho \equiv q+b=(2 \pi)^{-\frac{1}{2}} \int\left[1+e^{\beta(\alpha+z \sqrt{q})}\right]^{-1} e^{-\frac{1}{2} z^{2}} d z \\
q=(2 \pi)^{-\frac{1}{2}} \int\left[1+e^{\beta(\alpha+z \sqrt{q})}\right]^{-2} e^{-\frac{1}{2} z^{2}} d z .
\end{gathered}
$$

As can be seen following the line of $[9,10]$ the physical significance of $\rho$ and $q$ is:

$$
\rho=\overline{\langle\tau\rangle} \quad q=\overline{\langle\tau\rangle^{2}},
$$

where, following the notations of [2], a bar denotes the average over quenched disorder.

For $\beta=0$ we have $\rho=\frac{1}{2}$ and $q=\frac{1}{4}$, as we expect from their physical significance. In the high temperature regime we can solve (23) by expansion in powers of $\beta$, and this yields:

$$
\begin{gathered}
\rho=\frac{1}{2}+\frac{1}{4} \beta g+\frac{1}{32} \beta^{2} \\
q=\frac{1}{4}+\frac{1}{4} \beta g+\frac{1}{16}\left(\frac{3}{4}+g^{2}\right) \beta^{2} .
\end{gathered}
$$

\section{Low temperature results}

At zero temperature we can perform a detailed analytic study of saddle point equations. In this limit eq. (23) leads to:

$$
\begin{gathered}
q=(2 \pi)^{-\frac{1}{2}} \int_{-\infty}^{\alpha / \sqrt{q}} e^{-\frac{1}{2} z^{2}} d z \equiv \operatorname{erf}\left(\frac{\alpha}{\sqrt{q}}\right) \\
\gamma_{0} \equiv \lim _{T \rightarrow 0} \beta(\rho-q)=(2 \pi q)^{-\frac{1}{2}} e^{-\frac{\alpha^{2}}{2 q}},
\end{gathered}
$$

the equation for $\rho$ is the same as that for $q$ indeed for $T \rightarrow 0$ we have $\rho=q+\gamma T$ and thus $\alpha=g+\frac{1}{2} \gamma_{0}$.

For $g<0$ there always is a solution of (25) with $q=0, \gamma_{0}=0$ and $\alpha \equiv g$. To look for solutions with $q \neq 0$ let us define $x \equiv \operatorname{erf}^{-1}(q)=\alpha / \sqrt{q}$, then we get:

$$
\rho=q=\operatorname{erf}(x)
$$




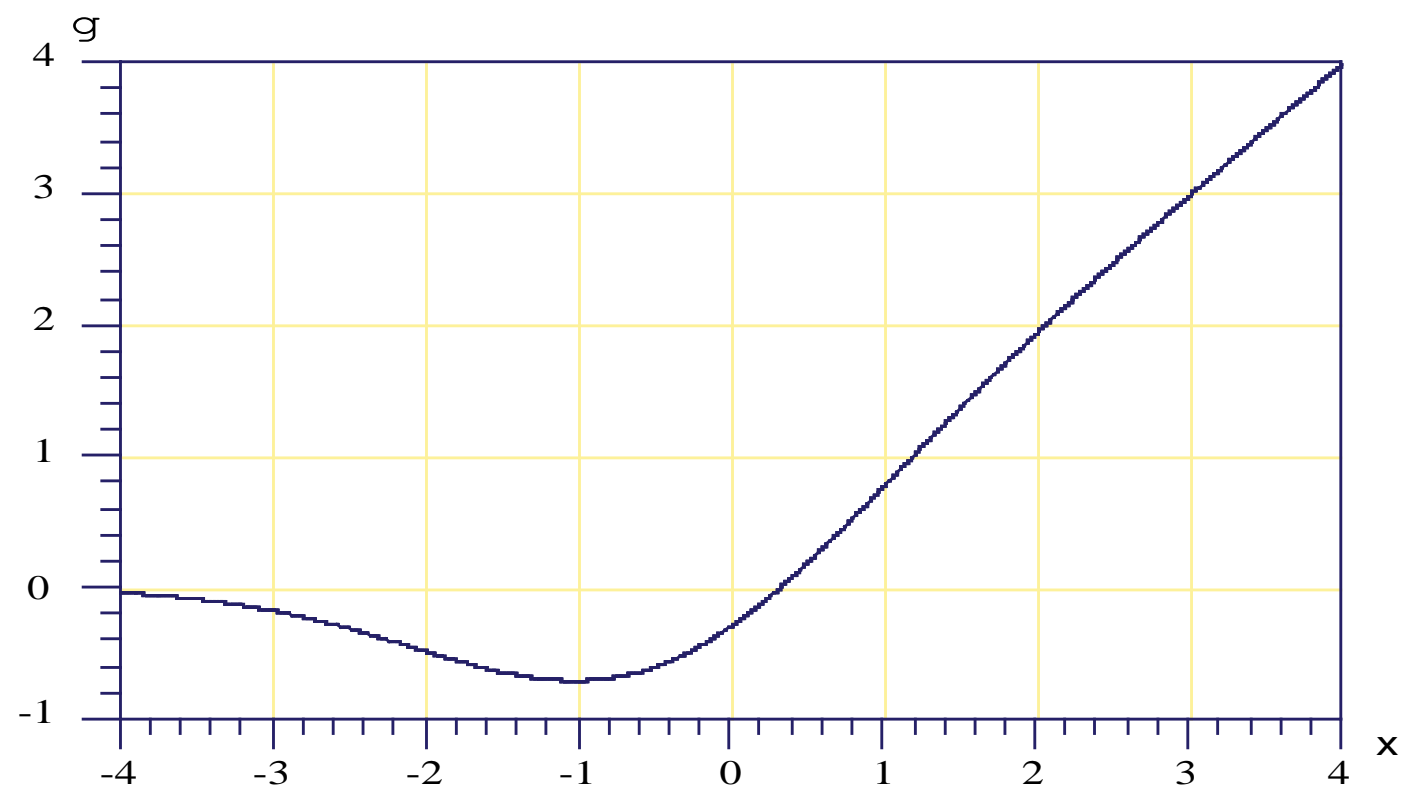

Figure 1: External field $g$ versus parameter $x\left(x \equiv \operatorname{erf}^{-1}(q)\right)$ as results from the last of (26). 


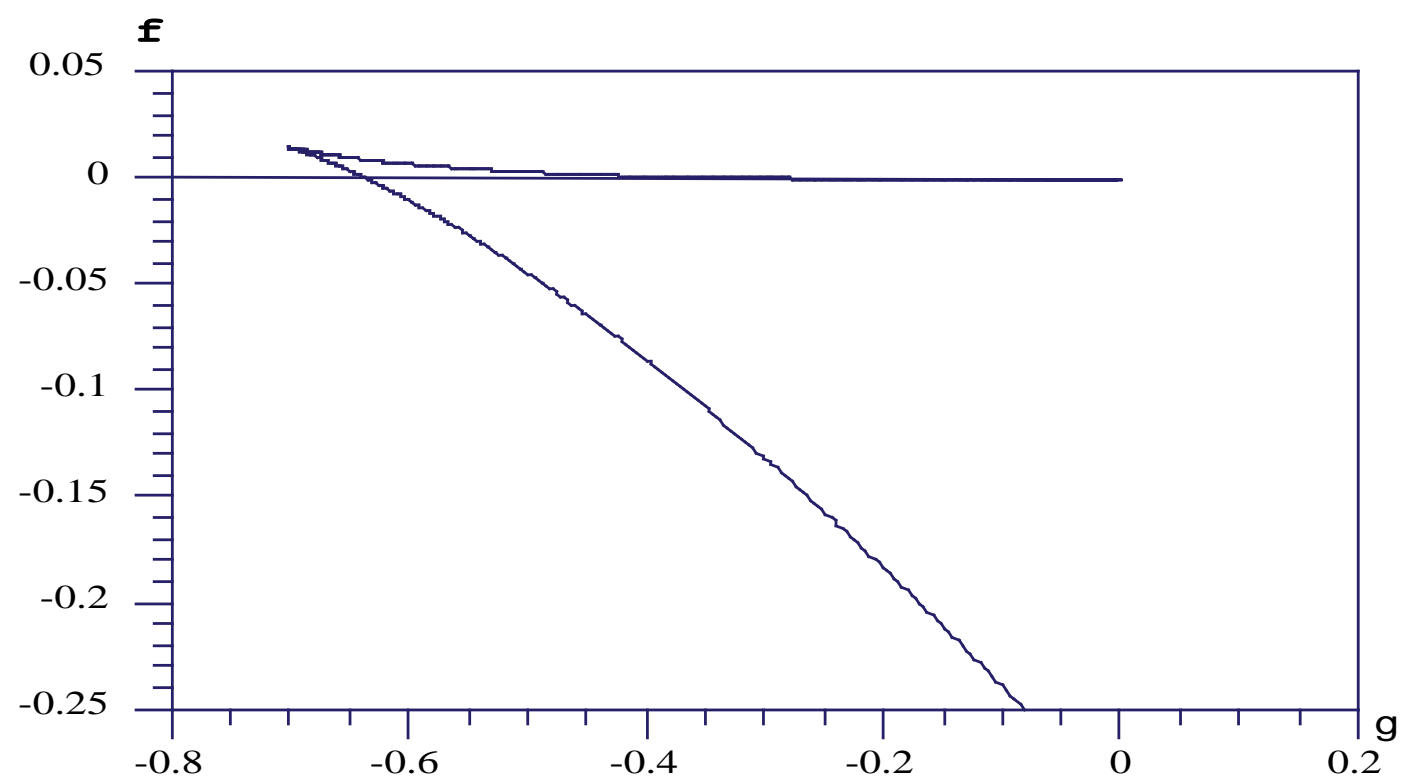

Figure 2: Free energy density versus external field at zero temperature in the replica symmetric solution. The transition point is where the two lower branches cross each-other. The higher branch is an unstable solution.

$$
\begin{gathered}
\gamma_{0}=[2 \pi \operatorname{erf}(x)]^{-\frac{1}{2}} e^{-\frac{1}{2} x^{2}} \\
\alpha=x \sqrt{\operatorname{erf}(x)} \\
g=\alpha-\frac{1}{2} \gamma_{0}=x \sqrt{\operatorname{erf}(x)}-\frac{1}{2}[2 \pi \operatorname{erf}(x)]^{-\frac{1}{2}} e^{-\frac{1}{2} x^{2}},
\end{gathered}
$$

we can therefore express all relevant quantities as functions of parameter $x$. From the last of (26)'s (see fig. 1) we can see that each value of $g$ in the range $-0.70242<g<0$ corresponds to two values of $x$ both lower than 0.30859 . Summarizing, if $g<-0.70242$ or $g>0$ we have a single solution of (25) for each value of $g$, but if $-0.70242<g<0$ we have three solutions and in order to pick-up the physical one we have to impose the continuity of the free energy density as a function of external field, see fig. 2. We thus find a first-order phase transition in the point $g_{0}=-0.63633$ where the two lower branches of $f$ cross each-other, the transition point being determined by the condition $\gamma_{0}\left(g_{0}\right)=-g_{0}$. We show in fig. 3 and $4 q(=\rho)$ and $\gamma_{0}$ as functions of external field. As expected both are discontinuous on the transition point. 


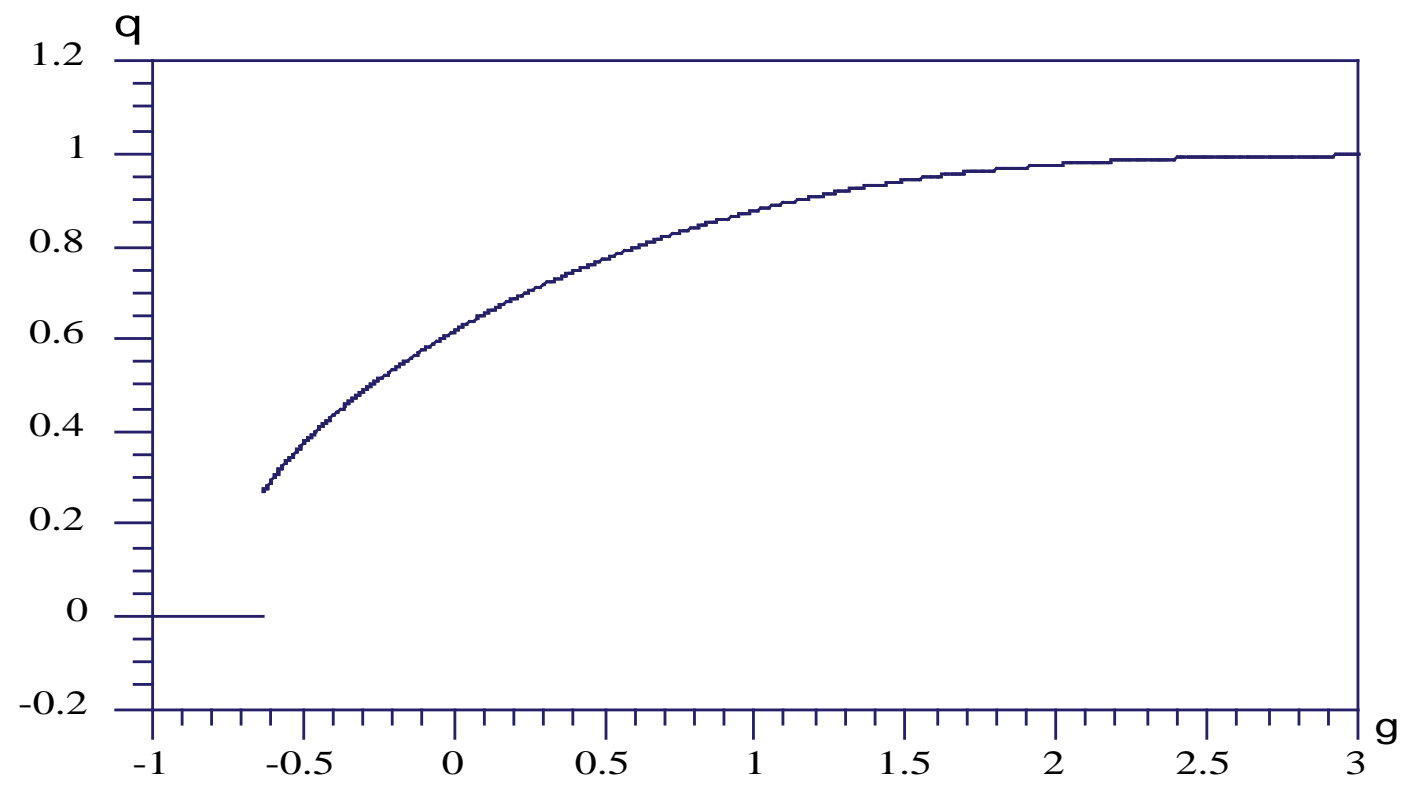

Figure 3: Replica symmetric order parameter $q(=\rho=\overline{\langle\tau\rangle})$ at $T=0$ as a function of external field.

Next we look to thermodynamical functions, the internal energy and entropy densities are given by:

$$
\begin{gathered}
u=-\left(g+\gamma_{0}\right) q \\
s=-\frac{1}{4} \gamma_{0}^{2} .
\end{gathered}
$$

The entropy (27) is negative in the range $g>g_{0}$ where $\gamma_{0}$ is different from zero, so we should expect the Replica Symmetry to be broken in this region. We stress that, differently from SK case, we have a region in which the Replica Symmetric Solution remains physical down to zero temperature. The maximum absolute value of the zero temperature entropy is at $g=g_{0}^{+}$ where it takes the value 0.101 and it strongly decreases for higher values of $g$ (e.g. at $g=1 \quad s=-0.011)$.

For $\beta \gg 1$ the solution of (23) can behave in two different ways. In the range $g<g_{0}$, where $q_{0}\left(\left.\equiv q(T ; g)\right|_{T=0}\right)$ and $\gamma_{0}(g)$ are identically zero, we find that all their temperature derivatives vanish for $T \rightarrow 0, q$ vanishes as $e^{\beta g}(g<0)$ and $\gamma(\equiv \beta(\rho-q))$ as $\beta e^{\beta g}$. Otherwise if $g>g_{0}$, they depend 


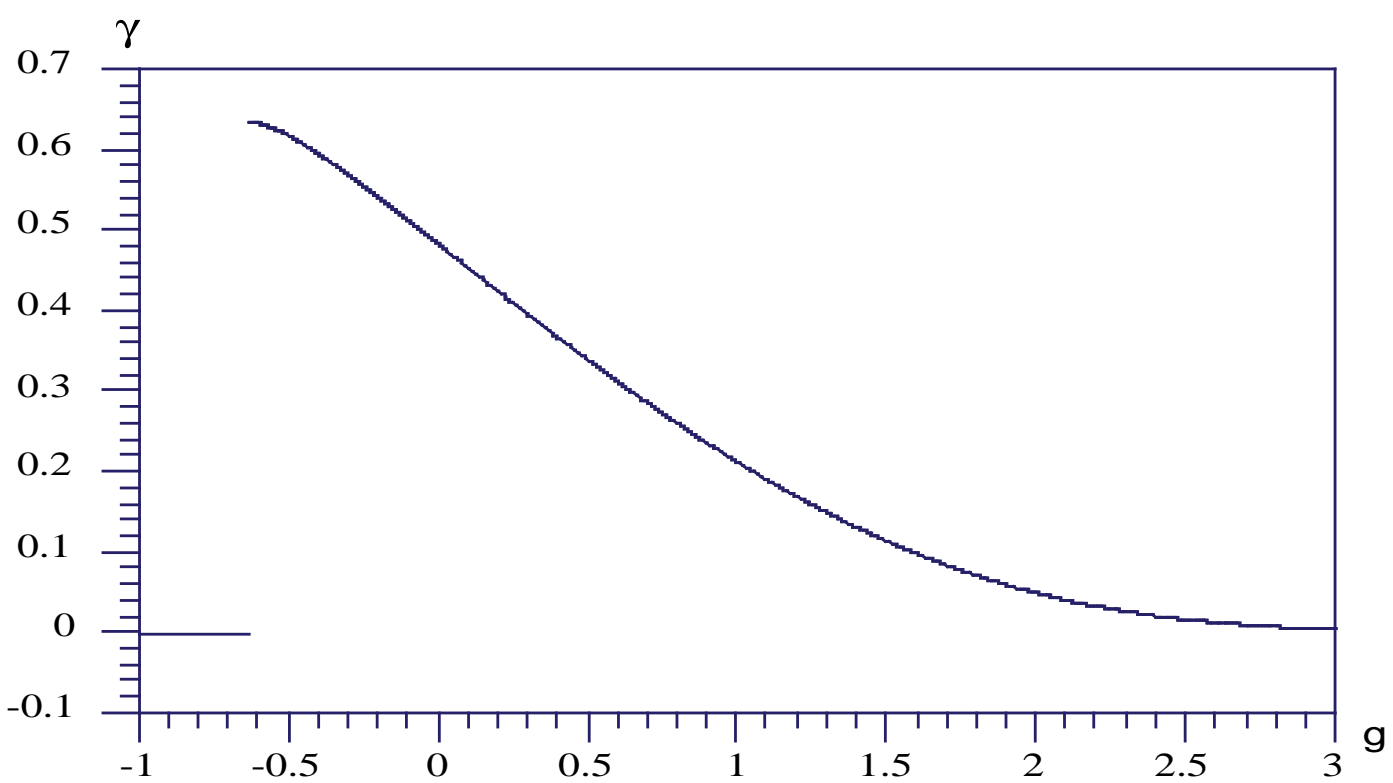

Figure 4: Behaviour of $\gamma_{0}\left(=\lim _{T \rightarrow 0} \beta \overline{\left(\left\langle\tau^{2}\right\rangle-\langle\tau\rangle^{2}\right)}\right)$, in the replica symmetric solution, as a function of external field. 


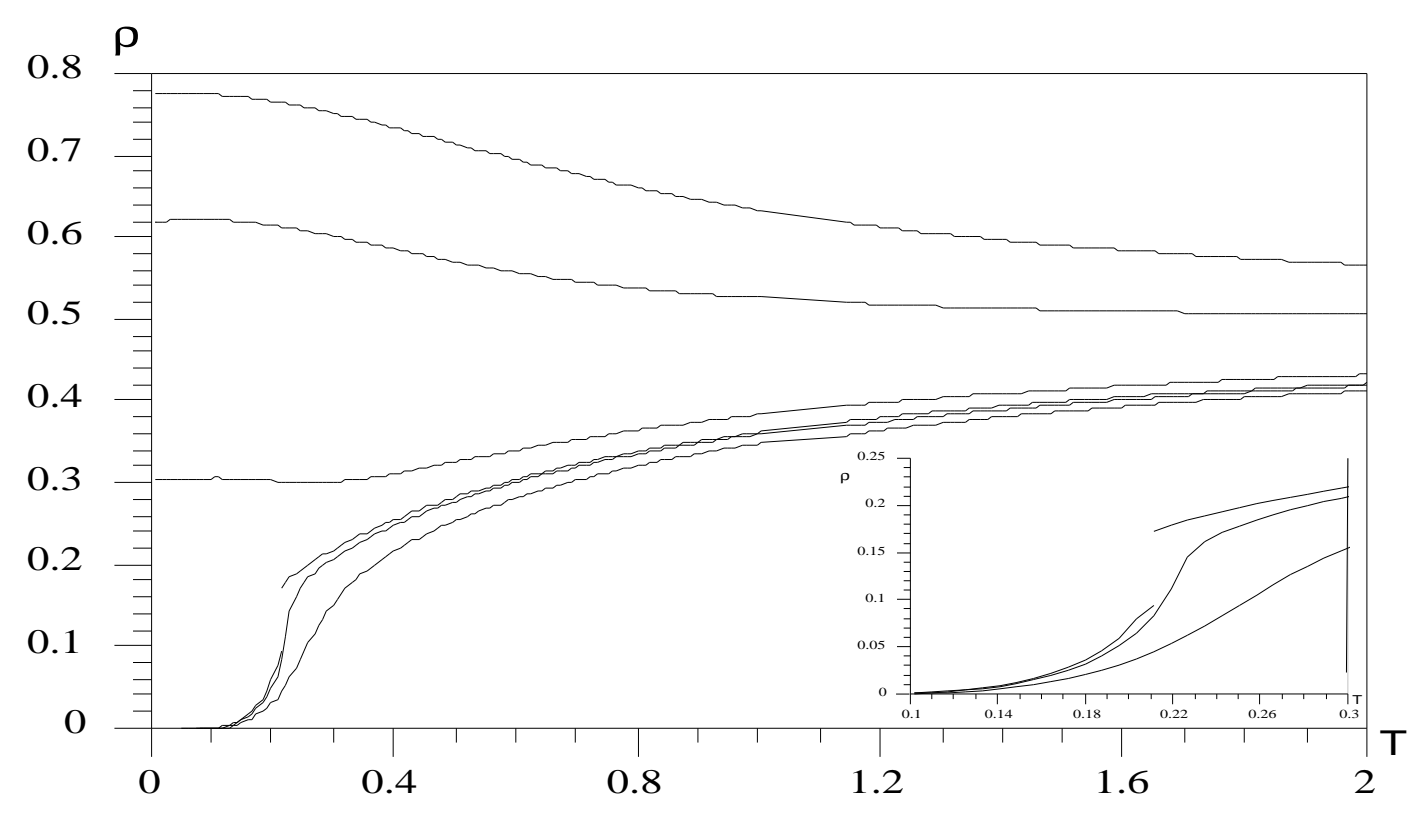

Figure 5: Replica symmetric order parameter $\rho$ as function of temperature for several values of external field. From top to bottom the values of $g$ are $0.5,0,-0.6,-0.68,-0.7$ and -0.75 . The embedded picture represents a magnification of the region around the transition.

linearly on $T$, indeed we get:

$$
\begin{gathered}
q=q_{0}-\frac{q_{0} \gamma_{0}\left(q_{0}+\frac{1}{2} \gamma_{0} \alpha_{0}\right)}{\left(q_{0}+\frac{1}{2} \gamma_{0} \alpha_{0}\right)^{2}+\frac{1}{4} \gamma_{0}\left(q_{0}-\alpha_{0}^{2}\right)} T \\
\gamma=\gamma_{0}+\frac{\frac{1}{2} \gamma_{0}^{2}\left(q_{0}-\alpha_{0}^{2}\right)}{\left(q_{0}+\frac{1}{2} \gamma_{0} \alpha_{0}\right)^{2}+\frac{1}{4} \gamma_{0}\left(q_{0}-\alpha_{0}^{2}\right)} T \\
\rho=q+\gamma T .
\end{gathered}
$$

We have also numerically solved (by iteration) equations (23) for several values of $T$ and $g$, results are plotted in fig. 5 and 6 . We find a line of first-order phase transitions in the phase diagram of fig. 7 . Such line ends in a second-order transition point for $T \simeq 0.22$ and $g \simeq-0.7$, as attested from fig. 8 where the linear response function $\chi=\partial \rho / \partial g$ (the "susceptibility") is plotted versus temperature for $g=-0.7$. 


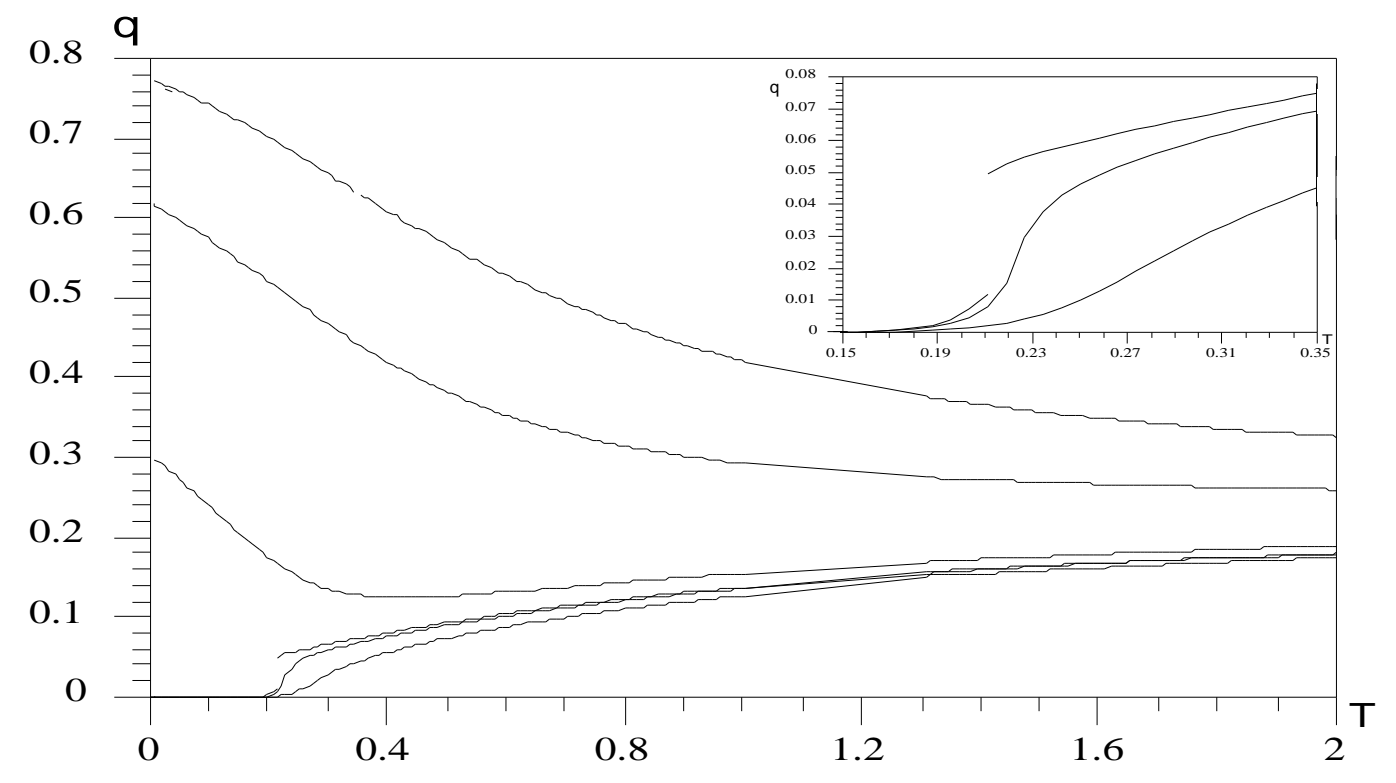

Figure 6: Replica symmetric order parameter $q$ as function of temperature for several values of external field. From top to bottom the values of $g$ are $0.5,0,-0.6,-0.68,-0.7$ and -0.75 . The embedded picture represents a magnification of the region around the transition. 


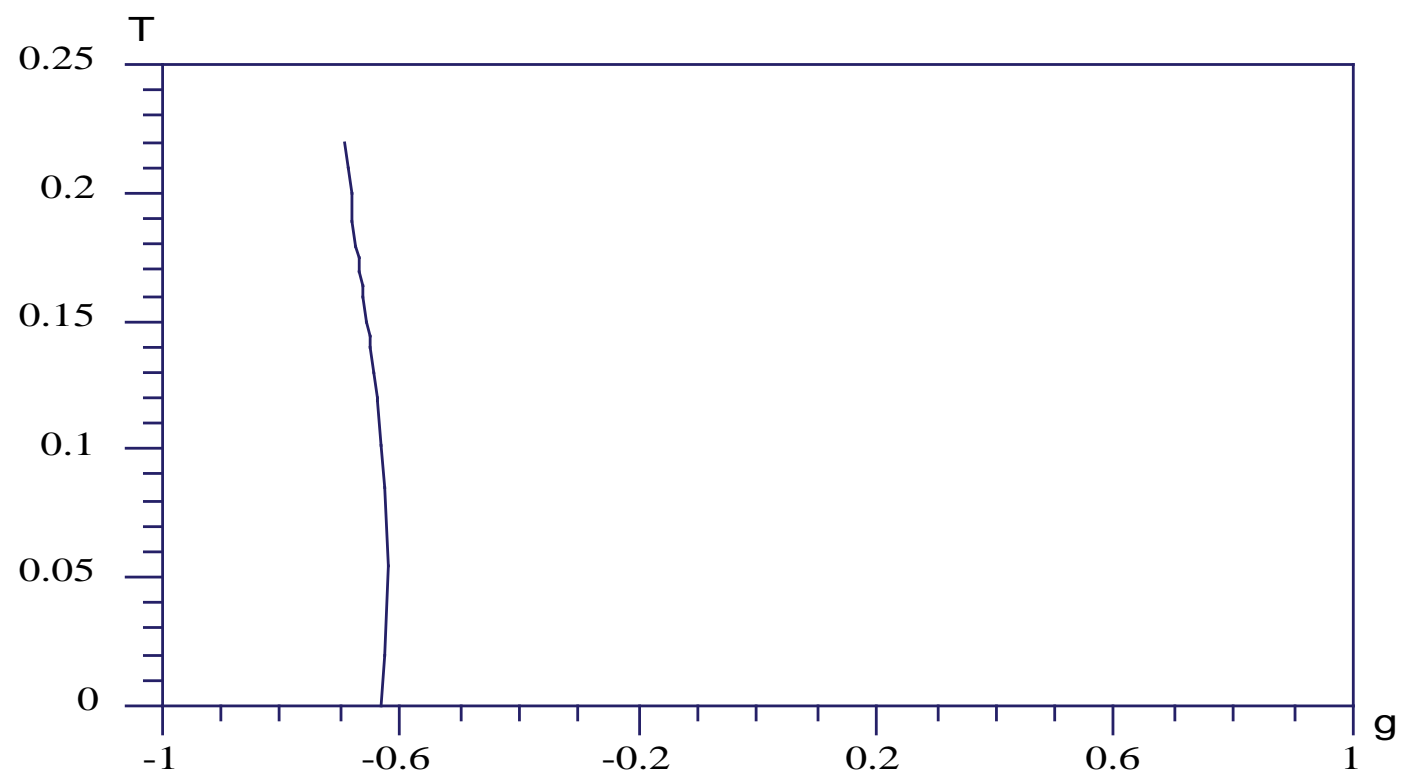

Figure 7: Phase diagram for the replica symmetric solution on the plane $(g ; T)$. A line of first order phase transitions ends with a second order transition point. 


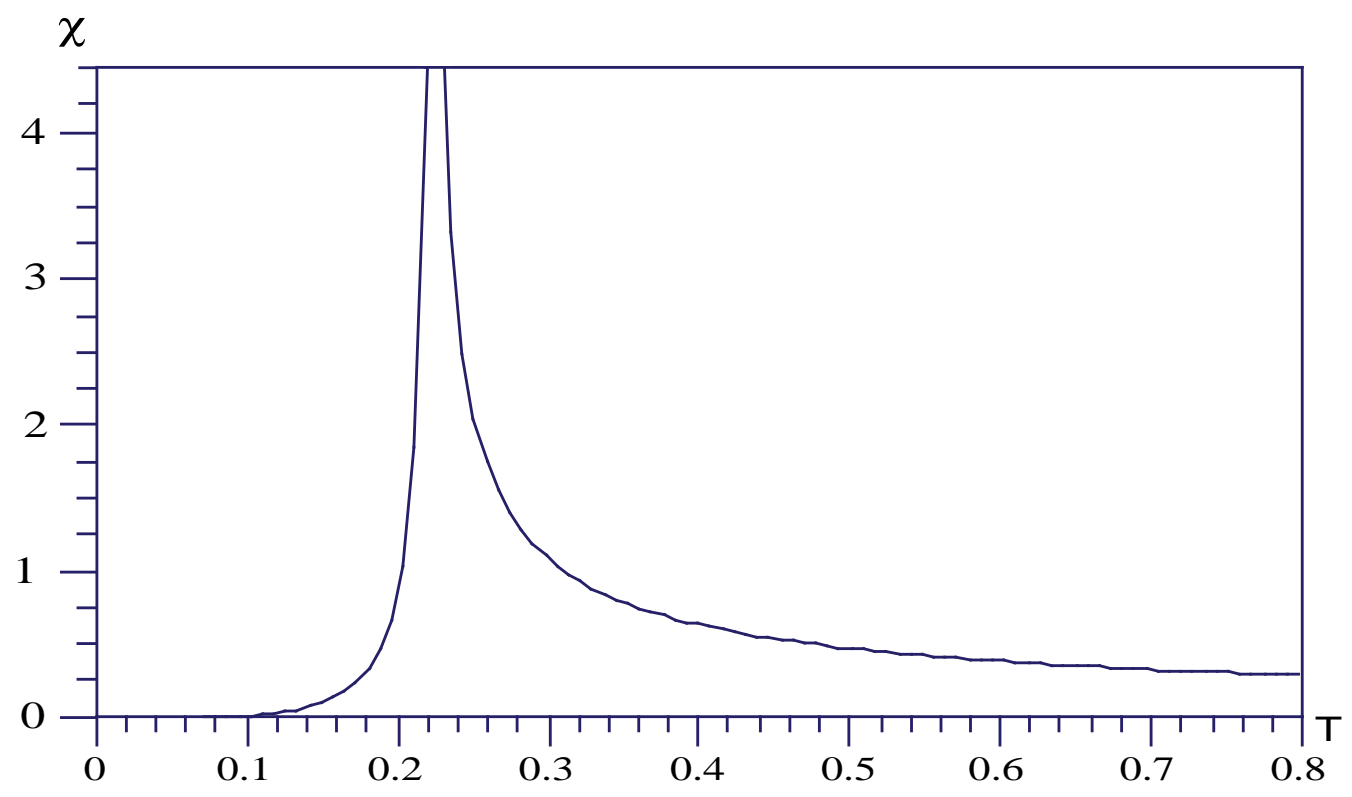

Figure 8: Linear Response Function $\chi=\partial \rho / \partial g$ ("susceptibility") as a function of temperature for $g=-0.7$. 


\section{$7 \quad$ Study of stability}

As we pointed out in the previous section the Replica Symmetric ansatz for the saddle point equations (21) can lead to unphysical results at low temperature. This should be considered as a signal[2] of Replica Symmetry Breaking (RSB). To check this conjecture we have to calculate the eigenvalues of the Hessian of (17), this will be done in this section following the line of ref. [12] but with two main differences. The first refers to the fact that we have $\bar{\phi}=0$ but the diagonal part of the matrix $Q_{a b}$ is different from zero and plays the same role as the magnetization vector of ref. $[9,10,12]$ where $\bar{J}=J_{0} \neq 0$. The second difference is that we never impose a priori $Q_{a b}$ to be a symmetric matrix. As a matter of fact the only term in $A[Q]$ depending on anti-symmetric part of $Q_{a b}$ is $\operatorname{Tr} Q^{2}$, its contribution could be integrated out and has no physical significance but allowing for its presence permits a more compact and elegant notation. We can indeed treat the diagonal and the off-diagonal part of $Q_{a b}$ in the same way. However we shall see that the saddle point is (as it should be) always a symmetric matrix and stable against anti-symmetric perturbation.

The Hessian of $A[Q]$ is:

$$
H_{a b}^{c d}=\frac{\partial^{2} A}{\partial Q_{a b} \partial Q_{c d}}=\frac{1}{2} \beta^{2} \delta_{a c} \delta_{b d}+\Gamma_{a b}^{c d},
$$

where we have set:

$$
\Gamma_{a b}^{c d}=\frac{1}{4} \beta^{4}\left[\left\langle\tau^{a} \tau^{b}\right\rangle_{Q}\left\langle\tau^{c} \tau^{d}\right\rangle_{Q}-\left\langle\tau^{a} \tau^{b} \tau^{c} \tau^{d}\right\rangle_{Q}\right]
$$

and the elements of $\Gamma$ have the following symmetries:

$$
\Gamma_{a b}^{c d}=\Gamma_{b a}^{c d}=\Gamma_{a b}^{d c}=\Gamma_{c d}^{a b}
$$

Remembering that $\tau^{2}=\tau$, and substituting the Replica Symmetric ansatz for the saddle point, we have the following different matrix elements for $\Gamma$ :

$$
\begin{gathered}
\Gamma_{a a}^{a a}=\frac{1}{4} \beta^{4}\left[\left\langle\tau^{a}\right\rangle_{Q}^{2}-\left\langle\tau^{a}\right\rangle_{Q}\right]=\frac{1}{4} \beta^{4}\left(\rho^{2}-\rho\right) \\
\Gamma_{a a}^{c c}=\frac{1}{4} \beta^{4}\left[\left\langle\tau^{a}\right\rangle_{Q}\left\langle\tau^{c}\right\rangle_{Q}-\left\langle\tau^{a} \tau^{c}\right\rangle_{Q}\right]=\frac{1}{4} \beta^{4}\left(\rho^{2}-q\right)
\end{gathered}
$$




$$
\begin{gathered}
\Gamma_{a b}^{a a}=\frac{1}{4} \beta^{4}\left[\left\langle\tau^{a} \tau^{b}\right\rangle_{Q}\left\langle\tau^{a}\right\rangle_{Q}-\left\langle\tau^{a} \tau^{b}\right\rangle_{Q}\right]=\frac{1}{4} \beta^{4}(q \rho-q) \\
\Gamma_{a b}^{c c}=\frac{1}{4} \beta^{4}\left[\left\langle\tau^{a} \tau^{b}\right\rangle_{Q}\left\langle\tau^{c}\right\rangle_{Q}-\left\langle\tau^{a} \tau^{b} \tau^{c}\right\rangle_{Q}\right]=\frac{1}{4} \beta^{4}\left(q \rho-\rho_{3}\right) \\
\Gamma_{a b}^{a b}=\frac{1}{4} \beta^{4}\left[\left\langle\tau^{a} \tau^{b}\right\rangle_{Q}^{2}-\left\langle\tau^{a} \tau^{b}\right\rangle_{Q}\right]=\frac{1}{4} \beta^{4}\left(q^{2}-q\right) \\
\Gamma_{a b}^{a d}=\frac{1}{4} \beta^{4}\left[\left\langle\tau^{a} \tau^{b}\right\rangle_{Q}\left\langle\tau^{a} \tau^{d}\right\rangle_{Q}-\left\langle\tau^{a} \tau^{b} \tau^{d}\right\rangle_{Q}\right]=\frac{1}{4} \beta^{4}\left(q^{2}-\rho_{3}\right) \\
\Gamma_{a b}^{c d}=\frac{1}{4} \beta^{4}\left[\left\langle\tau^{a} \tau^{b}\right\rangle_{Q}\left\langle\tau^{c} \tau^{d}\right\rangle_{Q}-\left\langle\tau^{a} \tau^{b} \tau^{c} \tau^{d}\right\rangle_{Q}\right]=\frac{1}{4} \beta^{4}\left(q^{2}-\rho_{4}\right),
\end{gathered}
$$

where explicitly written replica labels are different. In the $n \rightarrow 0$ limit, the required expectation values are:

$$
\begin{gathered}
\left\langle\tau^{a}\right\rangle_{Q^{*}}=Q_{a a}^{*}=\rho \\
\left\langle\tau^{a} \tau^{b}\right\rangle_{Q^{*}}=Q_{a b}^{*}=q \\
\left\langle\tau^{a} \tau^{b} \tau^{c}\right\rangle_{Q^{*}} \equiv \rho_{3}=(2 \pi)^{-\frac{1}{2}} \int\left[1+e^{-\beta(\alpha+z \sqrt{q})}\right]^{-3} e^{-\frac{1}{2} z^{2}} d z \\
\left\langle\tau^{a} \tau^{b} \tau^{c} \tau^{d}\right\rangle_{Q^{*}} \equiv \rho_{4}=(2 \pi)^{-\frac{1}{2}} \int\left[1+e^{-\beta(\alpha+z \sqrt{q})}\right]^{-4} e^{-\frac{1}{2} z^{2}} d z .
\end{gathered}
$$

The eigenvalues equation for the Hessian $H_{a b}^{c d}$ :

$$
\sum_{c, d} H_{a b}^{c d} \eta_{c d}=\lambda \eta_{a b}
$$

has, for general $n$, four classes of eigenvectors with no more than six distinct eigenvalues. Anti-symmetric eigenvectors $\left(\eta_{b a}=-\eta_{a b}\right)$ give the eigenvalue $\lambda_{0}=\frac{1}{2} \beta^{2}$ which (as we should expect) is always positive. Eigenvectors that are symmetric matrices, and invariant under the Replica Group, give two eigenvalues that, in the $n \rightarrow 0$ limit, are:

$\lambda_{1,2}=\frac{1}{2} \beta^{2}+\frac{1}{8} \beta^{4}\left(8 \rho_{3}-6 \rho_{4}-q-\rho\right) \pm \frac{1}{8} \beta^{4} \sqrt{\left(8 \rho_{3}-6 \rho_{4}-3 q+\rho\right)^{2}-16\left(\rho_{3}-q\right)^{2}}$.

Symmetric eigenvectors $\left(\eta_{b a}=\eta_{a b}\right)$ that are invariant under interchange of all but one of the replicas give, for general $n$, two more eigenvalues $\lambda_{3,4}$ that for 
$n \rightarrow 0$ reduce to the previous ones. There are finally symmetric eigenvectors that are invariant under interchange of all but two replicas, for $n \rightarrow 0$ these give rise to the eigenvalue:

$$
\lambda_{5}=\frac{1}{2} \beta^{2}\left[1+\beta^{2}\left(2 \rho_{3}-\rho_{4}-q\right)\right] .
$$

In the high temperature regime the eigenvalues $\lambda_{1}$ and $\lambda_{2}$ are found to be, in spite of the hermiticity of the Hessian matrix, complex conjugate. This should not be too surprising because we are working in a space where the norm is not positive definite $\left(\lim _{n \rightarrow 0} \frac{1}{n} \operatorname{Tr} Q^{2}=\rho^{2}-q^{2}\right)$. However, as could be seen considering the gaussian approximation to the integral in (18), we believe that the stability of the solution is determined by their (common) real part which is always positive. At lower temperature these eigenvalues are real and never negative, although they are found to vanish linearly on the second-order transition point. This means that this saddle-point is stable against Replica-Symmetric perturbation.

The eigenvalues $\lambda_{0} \lambda_{3}$ and $\lambda_{4}$ are not relevant and thus the stability of this solution against Replica-Symmetry-Breaking perturbations is determined by the eigenvalue $\lambda_{5}$. This eigenvalue is always real, to study its sign it is useful to define $\Lambda_{5}=2 T^{3} \lambda_{5}$, so we get:

$$
\Lambda_{5}=T-\frac{1}{8}(2 \pi q)^{-\frac{1}{2}} \int \frac{e^{-\frac{(\alpha+2 T u)^{2}}{2 q}}}{\cosh ^{4} u} d u .
$$

The low temperature limit then follows in a straightforward way and we find:

$$
\lim _{T \rightarrow 0} \Lambda_{5}=-\frac{1}{6}(2 \pi q)^{-\frac{1}{2}} e^{-\frac{\alpha^{2}}{2 q}}=-\frac{1}{6} \gamma_{0}
$$

It is therefore apparent that, if $g \geq g_{0}, \lambda_{5}$ must become negative at low enough temperatures. The Replica-Symmetric saddle point is unstable and the Replica Symmetry is spontaneously broken. We plot in figure 9 the behaviour of $\Lambda_{5}$ versus temperature for several values of $g$. The line of instability obtained by numerical evaluation is shown in figure 10, we stress the presence of a region in which the Replica Symmetry remains exact down to zero temperature. 


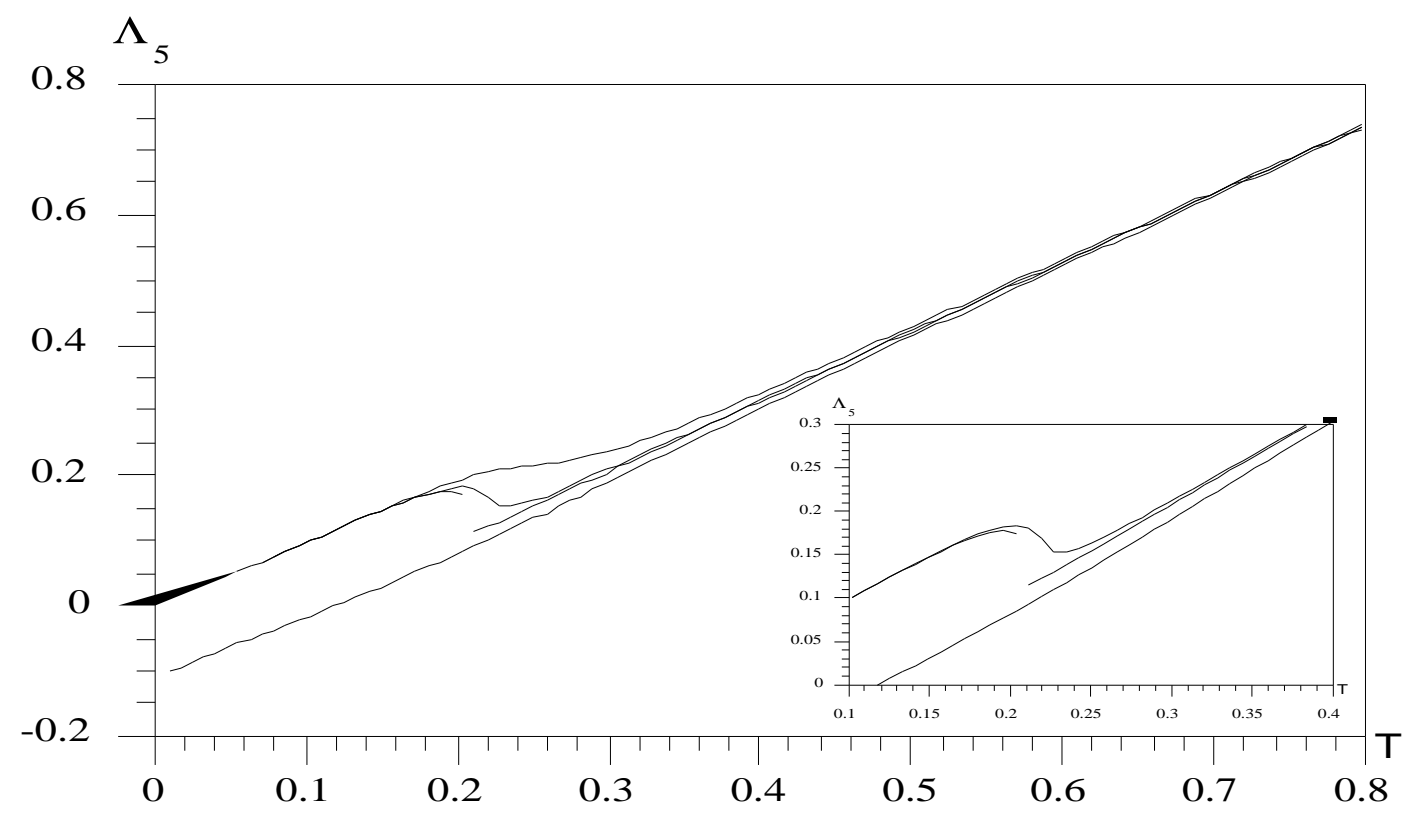

Figure 9: Behaviour of $\Lambda_{5}\left(=2 T^{3} \lambda_{5}\right)$ as a function of temperature for several values of external field. From bottom to top the values of $g$ are $-0.6,-0.68$, -0.7 and -0.75 . The embedded picture represents a magnification of the region around the transition. 


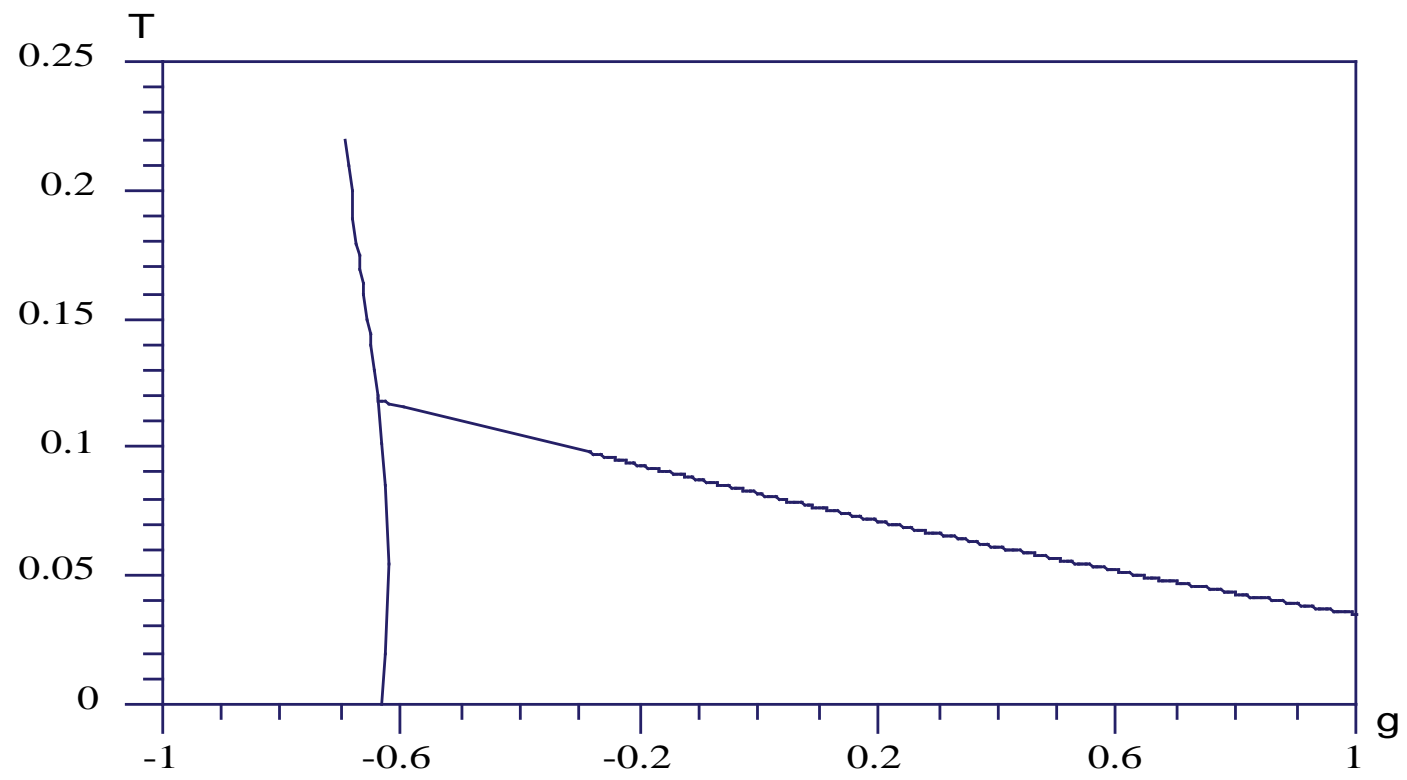

Figure 10: Limits of stability for the replica symmetric solution superimposed on its phase diagram. The region under the instability line is the Glassy Phase. 


\section{Zero temperature results with first order Replica Symmetry Breaking}

Once stated that the Replica Symmetry can be broken, we looked for saddle points that are not invariant under the Replica Group. As a first step we analyzed the zero temperature behaviour of the solution given by the ansatz proposed in ref. $[13,14]$. Let us divide the $n$ replicas in $\nu \equiv n / m$ groups, each of $m$ replicas, and take the matrix elements of $Q$ as follows:

$$
\begin{gathered}
Q_{a b}=\rho=q+d+b \quad \text { if } a=b \\
Q_{a b}=q_{1}=q+d \quad \text { if } a \neq b \quad \text { but } I(a / m)=I(b / m) \\
Q_{a b}=q_{0} \equiv q \quad \text { if } I(a / m) \neq I(b / m),
\end{gathered}
$$

where as usual[13] $I(x)=\min \{n \in N: n \geq x\}$. With this position we have $\operatorname{Tr} Q^{2}=n\left[\rho^{2}+(m-1) q_{1}^{2}+(n-m) q_{0}^{2}\right]$ and the effective Hamiltonian (16) reads:

$$
H_{Q}[\tau]=-\beta \alpha \sum_{a} \tau^{a}-\frac{1}{2} \beta^{2} q_{0}\left(\sum_{a} \tau^{a}\right)^{2}-\frac{1}{2} \beta^{2} d \sum_{l}\left(\sum_{k(a)=l} \tau^{a}\right)^{2}
$$

where $k(a)=I(a / m)$ and as previously we have set $\alpha=g+\frac{1}{2} \beta b$.

Using standard properties of Gaussian integrals, and setting $g_{\sigma}(x)=$ $(2 \pi \sigma)^{-\frac{1}{2}} e^{-\frac{x^{2}}{2 \sigma}}$, we find in the $n \rightarrow 0$ limit:

$$
f=\frac{1}{4} \beta\left[\rho^{2}+(m-1) q_{1}^{2}-m q_{0}^{2}\right]-\frac{1}{\beta m} \int g_{q_{0}}(z) \ln \left[I_{m}(\alpha+z ; d)\right] d z
$$

where:

$$
I_{m}(x ; d)=\int\left[1+e^{\beta(x+y)}\right]^{m} g_{d}(y) d y .
$$

Substituting the ansatz (32) in the saddle point equations (21) we find, in the $n \rightarrow 0$ limit, the following equations for $\rho, q_{1}$ and $q_{0}$ :

$$
\begin{aligned}
\rho & =\int g_{q_{0}}(z-\alpha) \frac{\int\left[1+e^{\beta(z+y)}\right]^{m-1} e^{\beta(z+y)} g_{d}(y) d y}{I_{m}(z ; d)} d z \\
q_{1} & =\int g_{q_{0}}(z-\alpha) \frac{\int\left[1+e^{\beta(z+y)}\right]^{m-2} e^{2 \beta(z+y)} g_{d}(y) d y}{I_{m}(z ; d)} d z
\end{aligned}
$$




$$
q_{0}=\int g_{q_{0}}(z-\alpha)\left[\frac{\int\left[1+e^{\beta(z+y)}\right]^{m-1} e^{\beta(z+y)} g_{d}(y) d y}{I_{m}(z ; d)}\right]^{2} d z .
$$

To decide if the correct saddle point has to be a maximum or a minimum of eq. (34) we note that $\lim _{n \rightarrow 0} \frac{1}{n} \operatorname{Tr} Q^{2}=\rho^{2}-(1-m) q_{1}^{2}-m q_{0}^{2}$ so, for $0 \leq m \leq 1$, we should expect the saddle point to be a minimum with respect to $\rho$ (diagonal parameter) and a maximum with respect to $q_{1}$ and $q_{0}$ (offdiagonal parameters). Because $m$ itself is a parameter for the off-diagonal part of $Q_{a b}$ we speculate that $f$ should be maximized with respect to it [2].

In order to work out the $T \rightarrow 0$ limit of eq. (36) let us consider the internal energy density:

$$
u=-g \rho-\frac{1}{2} \beta b\left(\rho+q_{1}\right)-\frac{1}{2} \beta m d\left(q_{0}+q_{1}\right) .
$$

To keep $u$ finite as $\beta \rightarrow \infty$ we set $b=\gamma T$ and $m=\mu T$, and we expect that $\gamma$ and $\mu$ remain finite as $T \rightarrow 0$, indeed in this limit eq. (36) leads to:

$$
\begin{gathered}
\gamma=\int \frac{g_{d}(z) g_{q_{0}}(\alpha+z)}{\operatorname{erf}\left(\frac{z}{\sqrt{d}}\right)+e^{-\mu\left(z-\frac{1}{2} \mu d\right)} \operatorname{erf}\left(\frac{\mu d-z}{\sqrt{d}}\right)} d z \\
\rho \equiv q+d=\int \frac{e^{-\mu\left(z-\frac{1}{2} \mu d\right)} \operatorname{erf}\left(\frac{\mu d-z}{\sqrt{d}}\right)}{\operatorname{erf}\left(\frac{z}{\sqrt{d}}\right)+e^{-\mu\left(z-\frac{1}{2} \mu d\right)} \operatorname{erf}\left(\frac{\mu d-z}{\sqrt{d}}\right)} g_{q_{0}}(\alpha+z) d z \\
q=\int\left[\frac{e^{-\mu\left(z-\frac{1}{2} \mu d\right)} \operatorname{erf}\left(\frac{\mu d-z}{\sqrt{d}}\right)}{\operatorname{erf}\left(\frac{z}{\sqrt{d}}\right)+e^{-\mu\left(z-\frac{1}{2} \mu d\right)} \operatorname{erf}\left(\frac{\mu d-z}{\sqrt{d}}\right)}\right]^{2} g_{q_{0}}(\alpha+z) d z .
\end{gathered}
$$

In the same limit the free energy density (34) then becomes:

$$
f=\frac{1}{4}[2 \gamma(q+d)+\mu d(2 q+d)]-\frac{1}{\mu} \int g_{q}(\alpha+z) \ln \left[I_{m}(z ; d)\right] d z,
$$

where:

$$
\lim _{T \rightarrow 0} I_{m}(z ; d)=\operatorname{erf}\left(\frac{z}{\sqrt{d}}\right)+e^{-\mu\left(z-\frac{1}{2} \mu d\right)} \operatorname{erf}\left(\frac{\mu d-z}{\sqrt{d}}\right) .
$$

Equations (37) have been numerically solved by iteration with $\mu$ held fixed, next the resulting free energy (38) has been maximized with respect to $\mu$ 


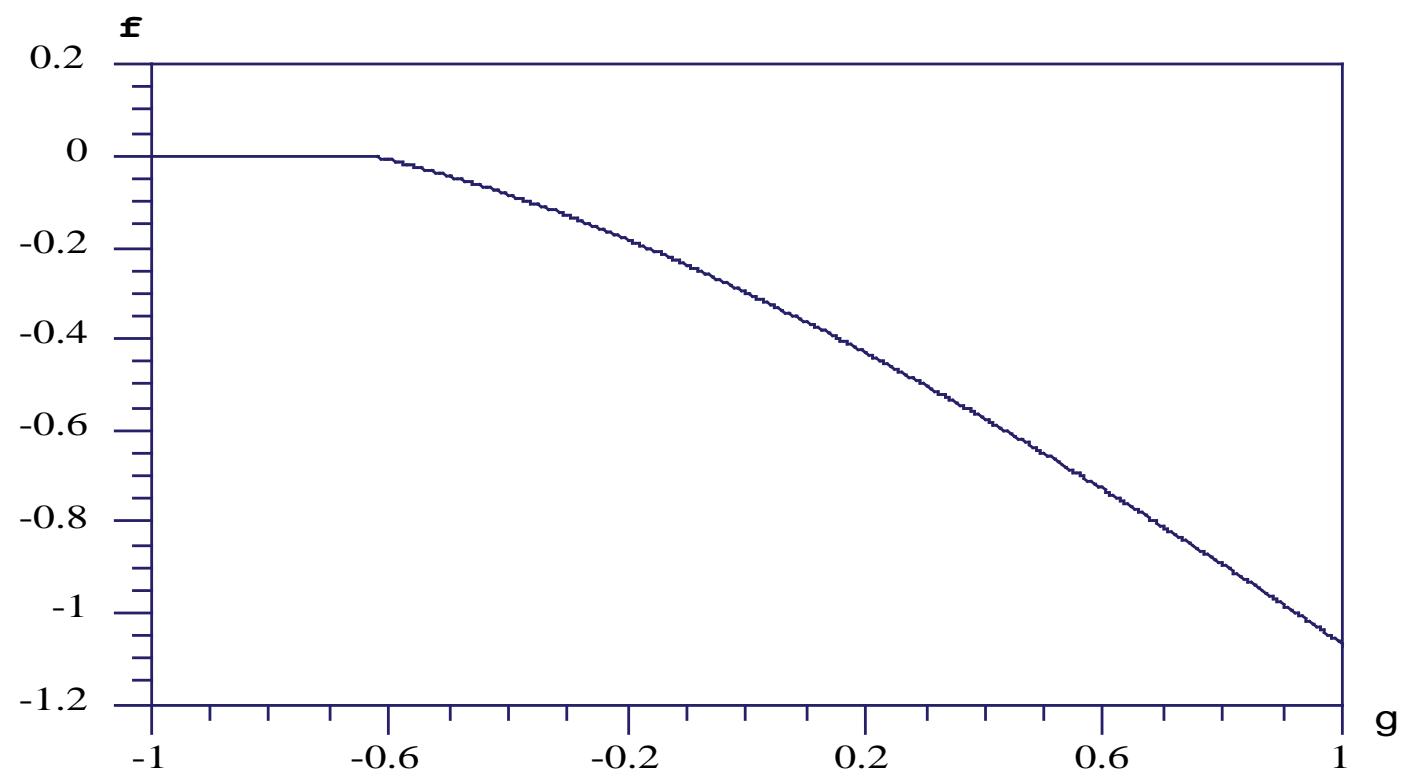

Figure 11: Free energy density versus external field at zero temperature with first order replica symmetry breaking. The first order transition point is at $g=-0.625$. 


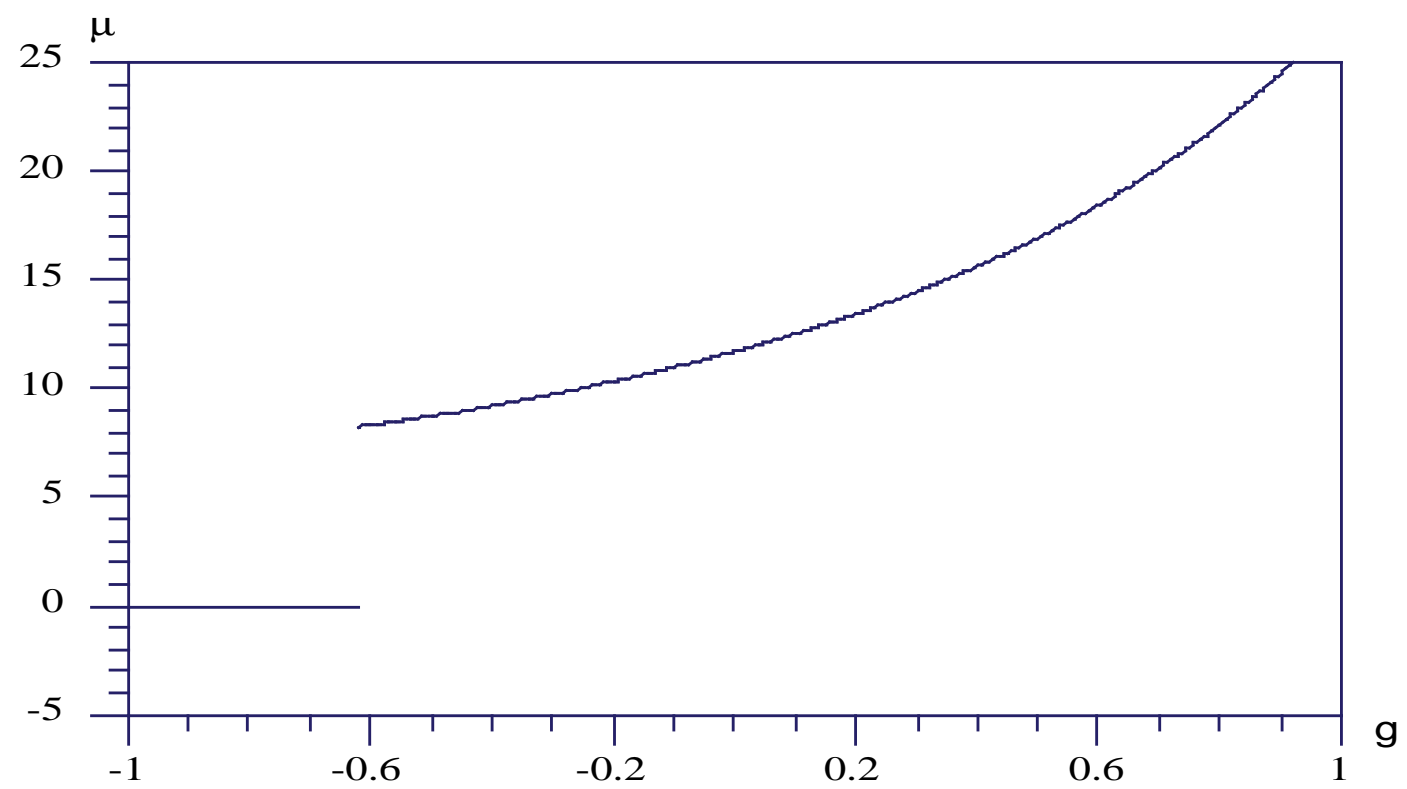

Figure 12: Behaviour of $\mu\left(=\lim _{T \rightarrow 0} \beta m\right)$ as a function of external field.

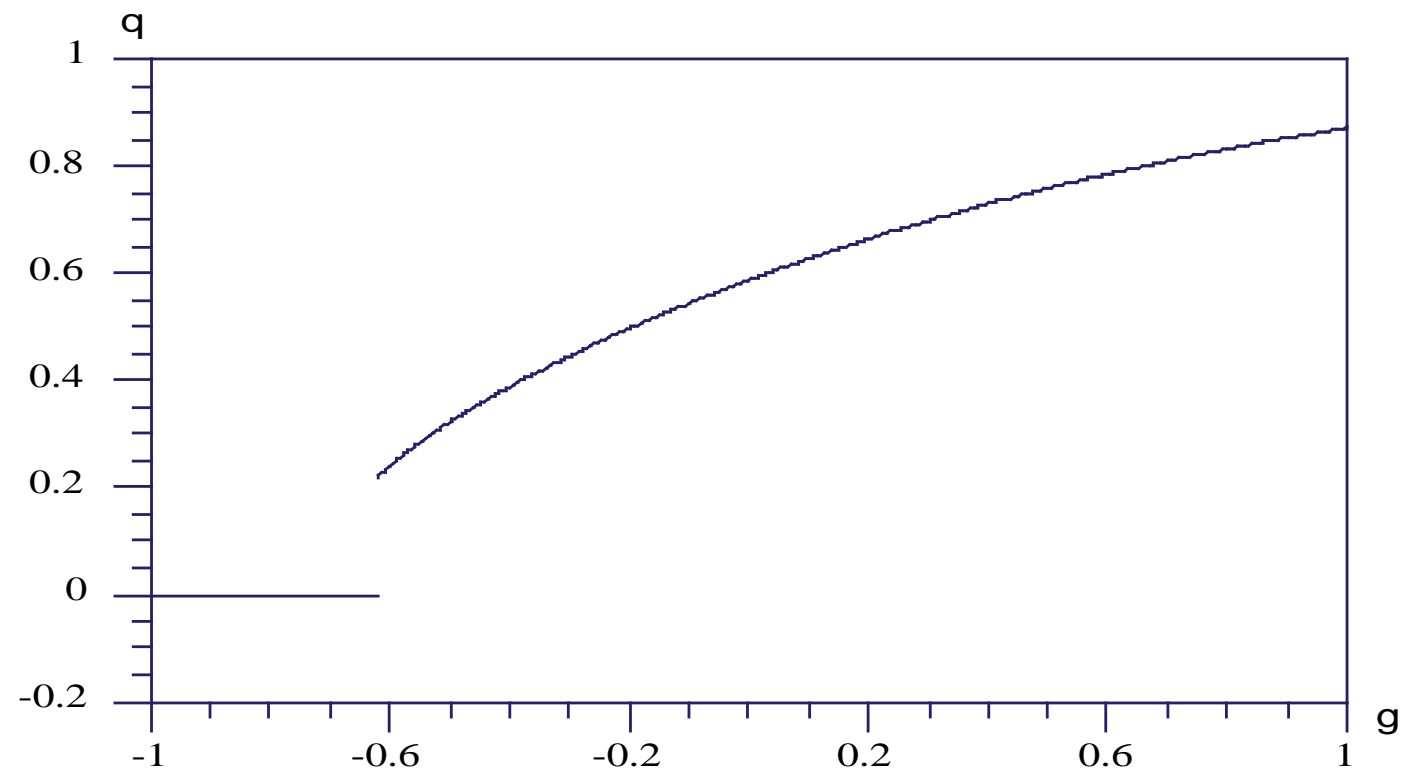

Figure 13: Solution with first order replica symmetry breaking: Off-diagonal order parameter $q\left(=q_{0}\right)$ at $T=0$ versus external field. 


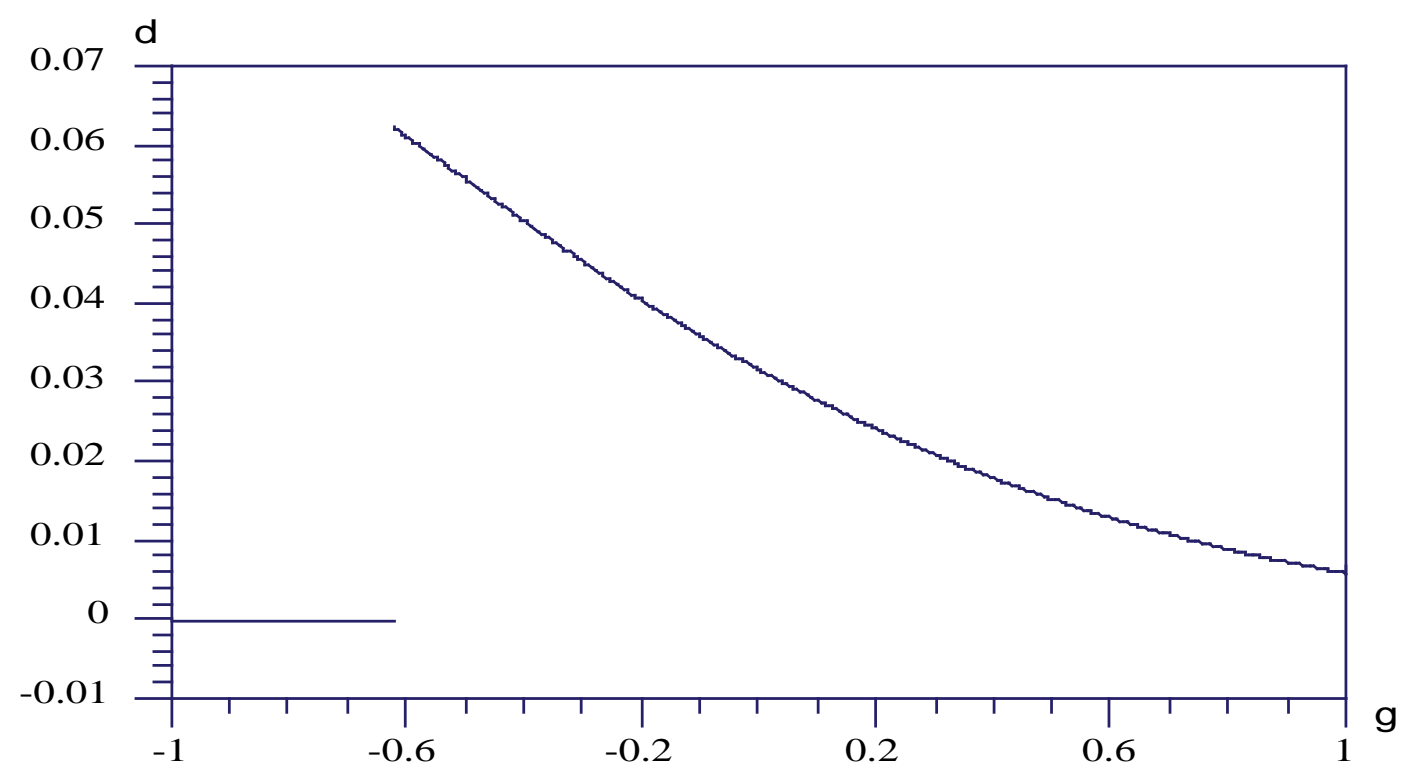

Figure 14: Solution with first order replica symmetry breaking: Symmetry breaking parameter $d\left(=q_{1}-q_{0}\right)$ as a function of external field at zero temperature. 


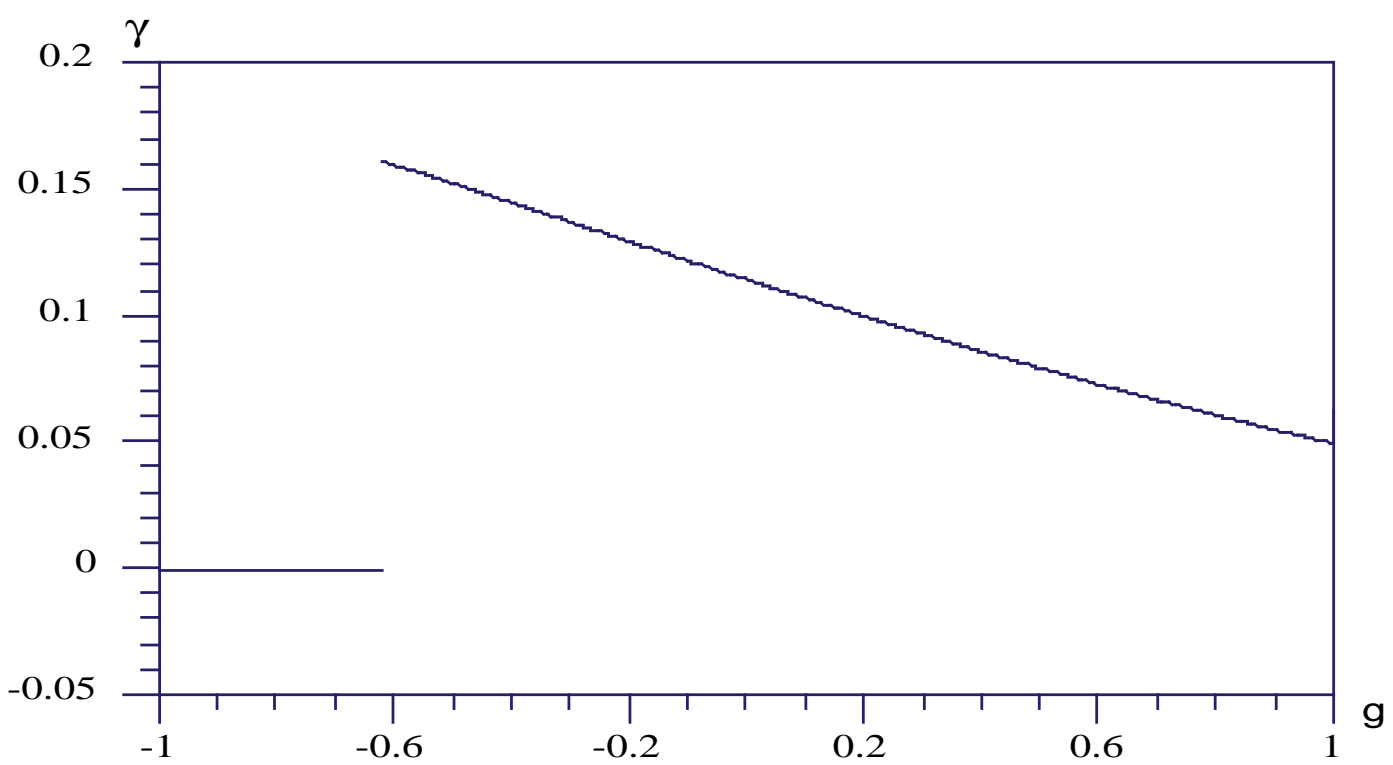

Figure 15: Solution with first order replica symmetry breaking: Behaviour of $\gamma\left(=\lim _{T \rightarrow 0} \beta\left(\rho-q_{1}\right)\right)$ as a function of external field.

using a standard IMSL routine. Such numerical solution of the saddle point problem gives the results plotted in figures 11-15 as functions of $g$. We note that the transition point is shifted from $g_{0}=-0.63633$ to $g_{0} \simeq-0.6250$ and the discontinuity of $q(0.27)$ is split in 0.28 for $\rho$ and 0.22 for $q_{0}$; we also note that the values of $\gamma$ are about a factor four smaller than those obtained in the Replica Symmetric approximation.

\section{Discussion conclusions and outlook}

In this paper we have investigated the thermodynamical properties of a lattice-gas model with infinite-ranged random interactions. We have discussed its relation with SK model and showed that the averaged thermodynamical functions of the two systems are not directly related to each other.

In SK case the zero-field Hamiltonian has a global $Z_{2}$ symmetry and all thermodynamical functions are either even or odd in the external field. SK model can have a first-order phase transition, as a function of magnetic field, 
only if a strong enough ferromagnetic part (i.e. a non-zero mean) is added to the random coupling, such a transition is indeed related to the breaking of the global $Z_{2}$ symmetry induced (as in the homogeneous case) by a ferromagnetic coupling. Moreover, at each value of external field, SK's Replica Symmetric Solution always becomes unstable for low enough temperature.

It is apparent how our picture is different from the usual one. We have considered the case of purely (zero-mean) random interactions and our Hamiltonian has no $Z_{2}$ symmetry. Nevertheless the Replica Symmetric Solution of our system exhibits a line of first-order phase transition points ending with a second-order transition point. This feature is robust to Replica Symmetry Breaking, indeed the second-order transition point lies outside the unstable region and we only expect that the transition line should be deflected on the instability boundary. Two phases co-exist along the transition line and, as we have stressed, in one of them the Replica Symmetry is exact down to zero temperature. We think that this is a consequence of the fact that in this phase the system should behave like an homogeneous one.

To have a physical interpretation of the obtained phase diagram it is useful to return to a more conventional framework for lattice gases. The effective Hamiltonian (1) describes a system of particles, in a discrete space, interacting with a two body potential $\phi_{k l}$. In this context $g$ is closely related to the chemical potential and the thermodynamic potential (4) is the negative of the pressure. The parameters $\rho$ and $q$ are respectively the quenched averages of particle density and of its square. When a first-order transition occurs there are two coexisting phases, the low-density one is to be interpreted as the gas phase, while the other is the liquid one. In our model the gas phase is that which is replica-stable at all temperatures. On the other hand, at sufficiently low temperatures, the Replica Symmetry breaks inside the liquid phase giving up to a glassy state. A part of the line of first-order transitions may therefore be interpreted as glass in equilibrium with its vapour.

We thus have a simple and soluble mean-field model accounting (in a conventional and so far well understood way) for both a liquid-gas transition and a glassy regime. This could be regarded as an intriguing paradigm for capturing the structural glass transition in the framework of replica theory.

There is a flaw in this. We have analyzed a model with quenched (random) disorder included by hand. Structural glasses instead do not necessarily have random interactions in their hamiltonians. In several recent publications (see e.g. $[4,5,6]$ ) the main effort was first to present a system, without 
random interactions, which behave in a glassy way, and next try to construct a disordered model which mimics the starting one. Here we have to go in the opposite direction. We have a good candidate of random system, the way of proposing a corresponding deterministic one will be presented elsewhere[15].

\section{Acknowledgments}

I am very grateful to Prof. Giorgio Parisi for the help he gave in this work with his suggestions and experience, and also for his careful and critical reading of the original manuscript.

I acknowledge Prof. Enzo Marinari for stimulating discussions and encouragement and also for his illuminating lectures; I thank Dr. Felix Ritort for useful discussions on various topics related to the subject of this work.

I wish acknowledge INFN, "Sezione di Roma Tor Vergata", for support received during this work.

\section{References}

[1] K. Huang: Statistical Mechanics (John Wiley \& Sons, 1963).

[2] M.Mezard, G.Parisi and M.A.Virasoro: Spin Glass Theory and beyond (World Scientific (Singapore), 1987).

[3] M.V. Tsodycs and M.V. Feigel'man: The Enhanced Storage Capacity in Neural Networks with Low Activity Level. Europhysics Letters 6(2), 101-105 (1988).

[4] J. P. Bouchaud and M. Mèzard: Self Induced Quenched Disorder: A Model for the Glass Transition. J. Physique I 4, 1109 (1994). cond-mat/9405075.

[5] E. Marinari, G. Parisi and F. Ritort: Replica field theory for deterministic models: I. 
Binary sequences with low autocorrelation.

J.Phys. A 27, 7615-7645 (1994).

hep-th/9405148.

[6] E. Marinari, G. Parisi and F. Ritort:

Replica field theory for deterministic models: II.

A non-random spin glass with glassy behaviour.

J.Phys. A 27, 7647-7668 (1994).

cond-mat/9406074.

[7] Francesco M. Russo:

On Lattice Gas Models For Disordered Systems

Physics Letters 239A, 17-20 (1998).

cond-mat/9710334.

[8] S. F. Edwards and P.W. Anderson:

Theory of spin glasses

J. Phys. F : Metal Phys., 5, 965 (1975).

[9] D. Sherrington and S. Kirkpatrick:

Solvable Model of a Spin-Glass

Phys. Rev. Lett. 35, 1792 (1975).

[10] S. Kirkpatrick and D. Sherrington:

Infinite-ranged models of spin glasses

Phys. Rev. B 17, 4384 (1978).

[11] B. Derrida:

Random-Energy Model:

an Exactly Solvable Model of Disordered Systems.

Phys. Rev. B 24, 2613 (1981).

[12] J.R.L. De Almeida and D.J. Thouless:

Stability of the Sherrington-Kirkpatrick solution of a spin glass model

J Phys. A 11, 983 (1978).

[13] G. Parisi:

Toward a mean field theory for spin glasses

Physics Letters 73A, 203 (1979). 
[14] G. Parisi:

A sequence of approximated solutions to the $S$-K model for spin glasses J. Phys. A : Math. Gen. 13, L115 (1980).

[15] Francesco M. Russo:

Work in progress. 